\title{
Development and Characterization of a Single Enzyme Linked Immunosorbent Assay for Detection of Antigen Specific IgG in Both Dogs and Cats
}

\author{
Brennan McKinney, Karen Blankenship and Kenneth Lee* \\ Stallergenes Greer, 639 Nuway Circle, Lenoir, North Carolina, USA
}

*Correspondence to: Kenneth W. Lee, PhD, Stallergenes Greer, 639 Nuway Circle, Lenoir, NC 28645; Tel: 828 759-7345, Fax: 828 754-7306; E-mail: ken.lee@ stallergenesgreer.com

Received: December 07, 2017; Accepted: December 21, 2017; Published: December 27, 2017

\begin{abstract}
The purpose of this study was to develop and characterize an enzyme linked immunosorbent assay for detection of antigen specific IgG in dogs and cats that might be used to document the clinical validity of $\operatorname{IgG}$ testing in these animals. The reactivity of multiple lots of affinity purified anti-IgG specific to the fc component of dog IgG or cat IgG, and subsequently biotinylated, were evaluated against varying dilutions of both cat and dog sera. An admixture of these anti-IgG-biotin antibodies $(25 \mathrm{nG} / \mathrm{mL}$ each) was optimized to yield similar responses with reactive calibrators for the two species. No substantial difference in responses between operators were noted; the average intra-assay \% CV for positive calibrators was calculated to be $5.7 \%$ (range $1.3 \%$ $12.9 \%$ ) and $8.3 \%$ (range $3.2 \%-19.2 \%$ ) for background responses. The average inter-assay variance for each of the operators was indistinguishable; the average \% CV was calculated to be $10.6 \%$ (range $7.0 \%-14.6 \%$ ). The intra-laboratory \% CV among reactive calibrators remained relatively constant at $11.8 \%$ (range $7.4 \%-13.1 \%$ ), while the background variance was calculated to be only slightly higher at $14.0 \%$. Evaluation of multiple samples from both cats and dogs, at a dilution of 1: 3000, against a panel of 24 environmental antigens ( 59 individual samples) and 24 food antigens (54 individual samples) demonstrate that IgG reactivity to all of these antigens is present in the majority of samples. Approximately $30 \%$ of the sample responses were within the lower range of detection ( 0 - 1000 EAU) and approximately $50 \%$ of all responses were in the mid-range (1001 - 3000 EAU) of detection, while the remaining $20 \%$ of sample response were in the upper range of detection (3001-4000 EAU) or beyond the limits of the assay. The results demonstrate the reproducibility and robustness of the assay and define its utility in detecting IgG specific for a number of different environmental and food antigens. Collectively, the results provide a foundation for future studies intended to address the issues associated with the validity of IgG testing (i.e. clinical sensitivity and specificity) for various antigens, especially those contained in food stuffs.
\end{abstract}

Key words: Dog IgG, Cat IgG, ELISA, Environmental Antigens, Food Antigens

\section{Introduction}

In recent years, information has been presented to document the advancements made in enzyme linked immunosorbent assays (ELISA) for detection of allergen specific IgE in companion animals [1-3]. Some of the commercially available assays have been well characterized and their functionality are continually monitored [2-7]. The utility of these IgE specific assays in confirming allergy diagnosis and providing a basis for selection of allergens to be included in immunotherapeutic regimes has been reasonably well established. In concert with these assays for detection of allergen specific IgE, ELISAs for detection antigen specific IgG have also been introduced [8-15] and substantial claims for the utility of these assays have been set forth in various promotional materials. A multitude of commercially available services are offered that range from monitoring allergen specific IgG following immunotherapy to those assays which purportedly define antigens that are involved with food hypersensitivity and/or intolerance. Unfortunately, very few studies have been published that characterize the assays used for these evaluations. Consequently, the clinical utility of these serum tests for detection of antigen specific IgG remains unclear. The results presented herein characterize a single ELISA that is designed to detect specific IgG to different environmental and food antigens in the sera of both dogs and cats that might provide a foundation for determining the validity of such testing.

\section{Materials and Methods}

\section{Buffers}

To maintain consistency of protocol and chemistry of assay components among assays for detection of allergen specific IgE, the IgG ELISA procedures used throughout this study mimics that of the previously characterized IgE macELISA for both dogs and cats. Thus, the buffers used were identical to those previously described [2-6] and include: a) well coating buffer: $0.05 \mathrm{M}$ sodium carbonate bicarbonate buffer, $\mathrm{pH}$ 9.6; b) wash buffer: phosphate buffered saline (PBS), pH 7.4, containing $0.05 \%$ Tween 20 , and $0.05 \%$ sodium azide; c) serum and reagent diluent buffer: PBS, pH 7.4, containing $1 \%$ fish gelatin, $0.05 \%$ Tween 20 and $0.05 \%$ sodium azide. 


\section{Sera}

Dog sera and cat sera samples used in this study were originally received for evaluation of allergen specific IgE and were shown to be non-reactive or borderline reactive using the respective macELISA. For assay development and characterization, a single dog sera pool was prepared by combining 41 individual samples while a similar cat sera pool was prepared using 57 individual cat sera samples. A sufficient volume of glycerin was added to each sera pool to yield a $50 \%$ solution; the volume of dog sera/glycerin pool equaled $66 \mathrm{~mL}$ whereas the cat sera/glycerin pool yielded $80 \mathrm{~mL}$. Sera pools and individual serum samples were stored at $-20^{\circ} \mathrm{C}$.

\section{Anti-IgG-Biotin Conjugate}

The anti-IgG-biotin second antibody conjugate for both dogs and cats was purchased from Jackson ImmunoResearch Laboratories, Inc (West Grove, PA). Three separate lots of affinity purified rabbit anti-dog IgG-biotin, Fc fragment specific (code number 304-065-008), and three separate lots of affinity purified goat anti-cat IgG-biotin, Fc fragment specific (code number 102-065-008), were evaluated. Each lot of antiIgG-biotin lyophilized material was reconstituted to a concentration of $1.0 \mathrm{mG} / \mathrm{mL}$ using an alkaline phosphatase stabilizing buffer (SigmaAldrich, St. Louis, MO) containing $50 \%$ glycerin; storage was at $-20^{\circ} \mathrm{C}$.

\section{Preparation of Coated Wells}

All allergen extracts used in this study were purchased from Stallergenes Greer (Lenoir, NC). Micro well flat bottom strip assemblies (Immulon $4 \mathrm{HBH}$, Thermo Electron Corporation, Waltham, MA) were used throughout and served as the solid phase for all ELISA evaluations. The twelve well strips were individually coated with the specified allergen extracts following a previously defined procedure $[2,4-6]$. Briefly, the individual extracts were diluted in bicarbonate buffer ( $\mathrm{pH}$ 9.6) and $100 \mu \mathrm{L}$ was added to each assigned well. Following overnight incubation at $4-8^{\circ} \mathrm{C}$, the wells were washed with PBS, blocked with $1 \%$ monoethanolamine ( $\mathrm{pH} 7.5$ ) then air dried and stored at $4-8^{\circ} \mathrm{C}$ in Ziploc bags until used.

\section{Allergen Panels}

Two separate allergen panels were used for evaluation of individual sera samples. The first panel was a 24 allergen composite that is routinely used for the proficiency evaluations of the various laboratories that routinely run the Stallergenes Greer macELISA for detection of allergen specific IgE, and is derived from the array of allergens that are included in the specific panels routinely evaluated in the various laboratories [4-6]. The composite allergen panel consists of 4 grasses, 6 weeds, 6 trees, 5 mites, and 3 fungi. The second antigen panel encompassed an array of 24 food antigens that included 12 meats, 6 grains, and 6 other specific foodstuffs.

\section{General ELISA Procedure for Sample Evaluations}

The prototype Enzyme Linked Immunosorbent Assay (ELISA) protocol used for detection of antigen specific immunoglobulins of various isotypes in different species of companion animals has been previously described [2, 4-6 ]. Briefly, $100 \mu \mathrm{L}$ of appropriately diluted sample is added to micro wells that had previously been coated with specifically defined allergens. Following an overnight incubation (1418 hours) at $4-8{ }^{\circ} \mathrm{C}$ in a humidified chamber, the wells are washed (2 complete aspirate/wash cycles using PBS wash solution), then $100 \mu \mathrm{L}$ of an appropriately diluted solution containing biotinylated anti-IgX (where $\mathrm{X}$ is equal to a species specific target isotype) second antibodies is added to each well. The wells are returned to the humidified chamber and incubation continued at room temperature $\left(20-25^{\circ} \mathrm{C}\right)$ for another 2 hours, and then they are washed (3 complete aspirate/wash cycles). Appropriately diluted Streptavidin-Alkaline Phosphatase Enzyme conjugate $(100 \mu \mathrm{L} /$ well $)$ is added and incubation at room temperature continued for 1 hour. Following a final wash step (4 complete aspirate/ wash cycles) $100 \mu \mathrm{L}$ of $p$-nitrophenylphosphate substrate ( $p$ NPP, Moss Substrates, Pasadena, Maryland) is added to each well and incubation continued for precisely 1 hour. Substrate development is then stopped by adding $50 \mu \mathrm{L}$ of $20 \mathrm{mM}$ cysteine to each well. Isotype specific antibody ( $\mathrm{IgX})$ reactivity to the antigens is estimated by determining the absorbance of each well measured at $405 \mathrm{nM}$ using an automated plate reader. All results are expressed as ELISA Absorbance Units (EAU) which are background corrected observed responses expressed as milli-absorbance.

Statistics: A coefficient of variation (\% CV) was calculated as the ratio of standard deviation and means of the responses observed for the solutions within different runs.

\section{Results}

Using a checkboard titration scheme, the reactivity of varying dilutions of sera containing grass specific $\operatorname{IgG}$ antibodies was determined using varying concentrations of separate lots of antiIgG-Biotin; evaluations of three separate lots of both anti-dog IgGbiotin and anti-cat IgG-biotin were completed by two separate operators. Because the results obtained by the two operators for all individual evaluations were indistinguishable, the data were treated as a single population. The results presented in Table 1 demonstrate that substantial grass reactivity is evident in the dog sera pool when evaluated with varying concentrations of anti-dog IgG-biotin antibodies. Although reduced in magnitude of response, the anti-dog IgG-biotin antibodies also yield substantial reactivity with varying dilutions of the cat sera pool. Similarly, substantial grass reactivity is evident in the cat sera pool when reacted with varying concentrations of anti-cat IgG-biotin antibodies (Table 2), and a reduced signal is also evident with this regent when evaluating varying dilutions of the dog sera pool. This dual reactivity demonstrated for the anti-dog IgGbiotin and the anti-cat IgG-biotin is likely a consequence of shared or cross reactive epitopes present on the IgG of the two species. To be expected, the magnitude of response reduces in direct proportion to the concentration of anti-IgG used as well as the dilution of sera evaluated. It is noteworthy that the signal evident without the presence of sera (i.e. background response) were indistinguishable between species and remains low regardless of the concentration of anti-IgGbiotin used. Among the various anti-IgG-biotin concentrations (10 $50 \mathrm{nG} / \mathrm{mL}$ ) that might be adopted for use in the assay the background responses remain indistinguishable. 
Table 1. Reactivity of varying concentrations of anti-DOG IgG-biotin when evaluated with varying dilutions of dog and cat sera pools.

\begin{tabular}{|c|c|c|c|c|c|c|c|}
\hline \multirow{2}{*}{$\begin{array}{c}\text { Anti-IgG-Biotin } \\
(\mathrm{nG} / \mathrm{mL})\end{array}$} & \multirow{2}{*}{$\begin{array}{r}N^{*} \\
(=12) \\
\end{array}$} & \multicolumn{5}{|c|}{ Dog Sera Dilution Factor } & \multirow[b]{2}{*}{$\underline{B G}$} \\
\hline & & 100 & 300 & 900 & 2700 & $\underline{8100}$ & \\
\hline \multirow[t]{2}{*}{2000} & Average & 3.786 & 3.732 & 3.575 & 2.769 & 1.269 & 0.119 \\
\hline & $\% \mathrm{CV}$ & 0.8 & 0.4 & 0.4 & 2.1 & 5.4 & 12.6 \\
\hline \multirow[t]{2}{*}{667} & Average & 3.798 & 3.761 & 3.604 & 2.696 & 1.195 & 0.104 \\
\hline & $\% \mathrm{CV}$ & 0.3 & 0.1 & 0.3 & 2.6 & 7.2 & 17.2 \\
\hline \multirow[t]{2}{*}{222} & Average & 3.834 & 3.795 & 3.606 & 2.527 & 1.079 & 0.077 \\
\hline & $\% \mathrm{CV}$ & 0.3 & 0.3 & 0.4 & 2.8 & 3.9 & 13.8 \\
\hline \multirow[t]{2}{*}{74} & Average & 3.854 & 3.787 & 3.517 & 2.125 & 0.878 & 0.076 \\
\hline & $\% \mathrm{CV}$ & 0.3 & 0.4 & 2.4 & 7.9 & 9.8 & 7.9 \\
\hline \multirow[t]{2}{*}{25} & Average & 3.832 & 3.688 & 3.016 & 1.423 & 0.571 & 0.094 \\
\hline & $\% \mathrm{CV}$ & 0.5 & 1.0 & 7.1 & 13.4 & 13.0 & 52.4 \\
\hline \multirow[t]{2}{*}{8} & Average & 3.618 & 3.071 & 1.736 & 0.757 & 0.310 & 0.069 \\
\hline & $\% \mathrm{CV}$ & 3.1 & 10.4 & 19.4 & 22.4 & 20.8 & 2.5 \\
\hline \multirow{2}{*}{$\begin{array}{c}\text { Anti-IgG-Biotin } \\
\text { (nG/mL) }\end{array}$} & $\mathrm{N}^{*}$ & \multicolumn{5}{|c|}{ Cat Sera Dilution Factor } & \\
\hline & $(=12)$ & 100 & $\underline{300}$ & $\underline{900}$ & $\underline{2700}$ & $\underline{8100}$ & $\underline{B G}$ \\
\hline \multirow[t]{2}{*}{2000} & Average & 3.763 & 3.712 & 3.532 & 2.646 & 1.176 & 0.175 \\
\hline & $\% \mathrm{CV}$ & 0.5 & 0.4 & 0.7 & 4.0 & 5.1 & 65.5 \\
\hline \multirow[t]{2}{*}{667} & Average & 3.774 & 3.674 & 3.253 & 1.683 & 0.676 & 0.100 \\
\hline & $\% \mathrm{CV}$ & 0.7 & 0.9 & 4.0 & 9.3 & 8.3 & 17.9 \\
\hline \multirow[t]{2}{*}{222} & Average & 3.718 & 3.343 & 1.910 & 0.798 & 0.324 & 0.073 \\
\hline & $\% \mathrm{CV}$ & 0.9 & 5.0 & 14.6 & 15.3 & 13.1 & 10.6 \\
\hline \multirow[t]{2}{*}{74} & Average & 3.168 & 1.907 & 0.889 & 0.378 & 0.179 & 0.075 \\
\hline & $\% \mathrm{CV}$ & 8.2 & 16.1 & 16.6 & 15.1 & 11.6 & 5.3 \\
\hline \multirow[t]{2}{*}{25} & Average & 1.581 & 0.786 & 0.380 & 0.184 & 0.112 & 0.074 \\
\hline & $\% \mathrm{CV}$ & 23.7 & 22.6 & 22.3 & 15.3 & 8.4 & 5.0 \\
\hline \multirow[t]{2}{*}{8} & Average & 0.705 & 0.366 & 0.190 & 0.113 & 0.084 & 0.070 \\
\hline & $\% \mathrm{CV}$ & 23.6 & 20.1 & 16.2 & 10.7 & 5.7 & 2.8 \\
\hline
\end{tabular}

* Three separate lots of anti-IgG dog-biotin were evaluated in duplicate by two separate technicians. 
Table 2. Reactivity of varying concentrations of anti-CAT IgG-biotin when evaluated with varying dilutions of cat and dog sera pools.

\begin{tabular}{|c|c|c|c|c|c|c|c|}
\hline \multirow{2}{*}{$\begin{array}{c}\text { Anti-IgG-Biotin } \\
(\mathrm{nG} / \mathrm{mL})\end{array}$} & \multirow{2}{*}{$\begin{array}{c}\begin{array}{c}N^{*} \\
(=12) \\
\end{array} \\
\end{array}$} & \multicolumn{5}{|c|}{ Cat Sera Dilution Factor } & \multirow[b]{2}{*}{$\underline{\mathrm{BG}}$} \\
\hline & & 100 & $\underline{300}$ & $\underline{900}$ & 2700 & $\underline{8100}$ & \\
\hline \multirow[t]{2}{*}{2000} & Average & 3.754 & 3.738 & 3.667 & 3.414 & 2.039 & 0.101 \\
\hline & $\% \mathrm{CV}$ & 0.4 & 0.3 & 0.4 & 0.7 & 5.5 & 10.0 \\
\hline \multirow[t]{2}{*}{667} & Average & 3.796 & 3.785 & 3.719 & 3.443 & 1.958 & 0.082 \\
\hline & $\% \mathrm{CV}$ & 0.4 & 0.5 & 0.3 & 1.0 & 5.6 & 5.9 \\
\hline \multirow[t]{2}{*}{222} & Average & 3.845 & 3.822 & 3.738 & 3.405 & 1.879 & 0.065 \\
\hline & $\% \mathrm{CV}$ & 0.2 & 0.2 & 0.2 & 1.2 & 5.6 & 8.0 \\
\hline \multirow[t]{2}{*}{74} & Average & 3.876 & 3.851 & 3.744 & 3.241 & 1.589 & 0.070 \\
\hline & $\% \mathrm{CV}$ & 0.4 & 0.5 & 0.4 & 3.0 & 8.5 & 2.3 \\
\hline \multirow[t]{2}{*}{25} & Average & 3.878 & 3.824 & 3.655 & 2.568 & 1.110 & 0.071 \\
\hline & $\% \mathrm{CV}$ & 0.4 & 0.3 & 1.0 & 6.6 & 10.8 & 3.4 \\
\hline \multirow[t]{2}{*}{8} & Average & 3.772 & 3.680 & 2.925 & 1.431 & 0.600 & 0.069 \\
\hline & $\% \mathrm{CV}$ & 0.8 & 1.6 & 9.4 & 14.1 & 15.8 & 2.5 \\
\hline \multirow{2}{*}{$\begin{array}{c}\text { Anti-IgG-Biotin } \\
(\mathrm{nG} / \mathrm{mL})\end{array}$} & $\mathrm{N}^{*}$ & \multicolumn{5}{|c|}{ Dog Sera Dilution Factor } & \\
\hline & $(=12)$ & $\underline{100}$ & $\underline{300}$ & $\underline{900}$ & $\underline{2700}$ & $\underline{8100}$ & $\underline{B G}$ \\
\hline \multirow[t]{2}{*}{2000} & Average & 3.751 & 3.668 & 3.338 & 1.883 & 0.787 & 0.109 \\
\hline & $\% \mathrm{CV}$ & 0.4 & 0.3 & 1.0 & 4.4 & 4.9 & 9.1 \\
\hline \multirow[t]{2}{*}{667} & Average & 3.760 & 3.629 & 2.906 & 1.349 & 0.537 & 0.085 \\
\hline & $\% \mathrm{CV}$ & 0.3 & 0.5 & 2.4 & 4.6 & 5.4 & 3.2 \\
\hline \multirow[t]{2}{*}{222} & Average & 3.716 & 3.303 & 1.743 & 0.705 & 0.292 & 0.068 \\
\hline & $\% \mathrm{CV}$ & 0.5 & 2.7 & 5.6 & 6.2 & 6.2 & 8.4 \\
\hline \multirow[t]{2}{*}{74} & Average & 3.368 & 1.910 & 0.804 & 0.336 & 0.161 & 0.071 \\
\hline & $\% \mathrm{CV}$ & 3.5 & 10.4 & 10.0 & 9.4 & 7.0 & 2.9 \\
\hline \multirow[t]{2}{*}{25} & Average & 1.857 & 0.821 & 0.354 & 0.167 & 0.102 & 0.070 \\
\hline & $\% \mathrm{CV}$ & 12.2 & 12.7 & 11.5 & 9.4 & 5.0 & 3.0 \\
\hline \multirow[t]{2}{*}{8} & Average & 0.745 & 0.353 & 0.174 & 0.104 & 0.079 & 0.069 \\
\hline & $\% \mathrm{CV}$ & 15.6 & 14.7 & 12.5 & 7.1 & 3.6 & 2.0 \\
\hline
\end{tabular}

*Three separate lots of anti-Cat IgG-biotin were evaluated in duplicate by two separate technicians. 
In light of the similarity of the reciprocal results for the anti-dog IgG-biotin and the anti-cat IgG-biotin combined with the consistency among manufactured lots of the reagents, it seems logical that a single assay might be developed for detection of antigen specific IgG in both dogs and cats. To this end, a single secondary anti-IgG antibody regent was prepared by mixing the previously evaluated lots of anti-dog IgGbiotin and anti-cat IgG-biotin. The concentration of each biotinylated anti-IgG was adjusted to $1 \mathrm{mG} / \mathrm{mL}$, then equal volumes of the separate reagents were mixed to yield a $1 \mathrm{mG} / \mathrm{mL}$ stock of anti-IgG-biotin reagent comprised of anti-dog IgG-biotin and anti-cat IgG-biotin with each at $500 \mu \mathrm{G} / \mathrm{mL}$.

The results presented in Table 3 document the reactivity of varying concentrations of this dual reactive reagent with varying dilutions of dog and cat sera pools. Clearly, substantial antigen specific IgG reactivity is demonstrable with either dog or cat sera when evaluated in ELISA using this reagent as the secondary detection antibody. It is likely that the affinity purified anti-IgG-biotin specific for each species contains approximately the same number of reactive molecules. Consequently, the increased signal evident with the cat sera dilutions is likely a result of a greater content of antigen specific IgG present in the cat sera pool. Never the less, we demonstrate that the signals decrease in direct proportion to the dilution of sera evaluated as well as the concentration of anti-IgG-biotin used; the character is consistent with assays of this sort $[16,17]$. The variances evident between different operators and among different assay runs is also consistent with previous results defined for assays of this sort [2, 4-6]. The overall average \% CV was calculated to be $13.0 \%$ (range, $2.1 \%-24.7 \%$ ) for dogs and $15.8 \%$ (range, $2.2 \%-29.4 \%$ ) for cats. The signals yielded with dog sera dilutions with an anti-dog IgGbiotin concentration of $25 \mathrm{nG} / \mathrm{mL}$ encompasses the potential range of reactivity and approximate the same order of magnitude of signals evident for the calibrators used in our assay for detection of allergen specific IgE in dog sera [2,4-6]. Consequently, the stock concentration of anti-IgG-biotin before dilution for use in an assay is adjusted to 25 $\mu \mathrm{G} / \mathrm{mL}$ (1000 X use concentration). For consistency between assays, the calibrators that are intended for use in the antigen specific IgG ELISA need to be constructed to approximate the signals evident in the ELISA for detection of allergen specific IgE.

Table 3. Reactivity of varying concentrations of admixtures of anti-dog IgG-biotin anti-cat IgG-biotin when evaluated with varying dilutions of cat and dog sera pools.

\begin{tabular}{|c|c|c|c|c|c|c|c|}
\hline \multirow{2}{*}{$\begin{array}{c}\text { Anti-lgG-Biotin* } \\
(\mathrm{nG} / \mathrm{mL})\end{array}$} & \multirow{2}{*}{$\begin{array}{r}\mathrm{N}^{\dagger} \\
(=12) \\
\end{array}$} & \multicolumn{5}{|c|}{ Dog Sera Dilution Factor } & \multirow[b]{2}{*}{$\underline{B G}$} \\
\hline & & 100 & $\underline{300}$ & $\underline{900}$ & 2700 & 8100 & \\
\hline \multirow[t]{2}{*}{50} & Average & 3.549 & 2.722 & 1.215 & 0.487 & 0.213 & 0.078 \\
\hline & $\% \mathrm{CV}$ & 2.1 & 9.9 & 11.7 & 9.7 & 8.2 & 4.9 \\
\hline \multirow[t]{2}{*}{25} & Average & 3.504 & 2.306 & 0.996 & 0.388 & 0.178 & 0.068 \\
\hline & $\% \mathrm{CV}$ & 5.3 & 17.4 & 17.1 & 15.6 & 11.8 & 9.3 \\
\hline \multirow[t]{2}{*}{12.5} & Average & 3.310 & 1.917 & 0.805 & 0.324 & 0.159 & 0.074 \\
\hline & $\% \mathrm{CV}$ & 22.8 & 24.7 & 20.8 & 14.4 & 3.4 & 0.0 \\
\hline \multirow{2}{*}{$\begin{array}{c}\text { Anti-IgG-Biotin } \\
(\mathrm{nG} / \mathrm{mL})\end{array}$} & $\mathrm{N}^{+}$ & \multicolumn{5}{|c|}{ Cat Sera Dilution Factor } & \\
\hline & $(=12)$ & 100 & 300 & 900 & $\underline{2700}$ & 8100 & $\underline{B G}$ \\
\hline \multirow[t]{2}{*}{50} & Average & 3.763 & 3.712 & 3.532 & 2.646 & 1.176 & 0.175 \\
\hline & $\% \mathrm{CV}$ & 2.2 & 9.1 & 12.5 & 13.2 & 10.4 & 7.4 \\
\hline \multirow[t]{2}{*}{25} & Average & 3.774 & 3.674 & 3.253 & 1.683 & 0.676 & 0.100 \\
\hline & $\% \mathrm{CV}$ & 4.0 & 17.1 & 20.1 & 18.7 & 15.5 & 9.6 \\
\hline \multirow[t]{2}{*}{12.5} & Average & 3.718 & 3.343 & 1.910 & 0.798 & 0.324 & 0.073 \\
\hline & $\% \mathrm{CV}$ & 11.7 & 26.9 & 29.4 & 27.0 & 19.5 & 3.7 \\
\hline
\end{tabular}

* Final concentration of anti-dog IgG-biotin and anti-cat IgG-biotin in equal concentration admixtures.

$\dagger$ Three separate lots of anti IgG-biotin admixtures evaluated in duplicate by two separate technicians. 
Because the ELISA for antigen specific IgG is designed to detect the respective antibodies in both dogs and cats, it is preferable that the calibrator system for this assay contain sera derived from both dogs and cats, and that the signal yielded with any dog sera are comparable to the signals observed with cat sera. To this end, we prepared a calibration system for the assay by preparing solutions containing appropriate mixtures of $\mathrm{dog}$ and cat sera. Before admixture, the appropriate dilution of each sera needed to yield comparable signals across a calibration range of 0 - 3500 EAU was empirically determined for each of the previously defined sera pools. For the current pools of cat and dogs sera that were defined in Tables 1-3, the mixture ratio has been defined to be 2.27. To attain the desired calibrator response for calibrator $\# 1\left(\mathrm{OD}_{405}=2.5-3.5\right)$ at this ratio using the current of dog and cat sera pools requires that the cat sera pool be at a 1: 1700 dilution, whereas the dog sera need be diluted 1: 750. To ensure equality of the signal magnitude for dogs and cats in the calibrator \#1 mixture, the cat sera pool was first diluted 1: 850 and the dog sera pool 1: 375 and evaluated separately. Admixture of equal volumes of the separately diluted sera yielded calibrator \# 1 to be used in the assay. Subsequent calibrator solutions (\# 2-5) were then prepared as a threefold serial dilution of the calibrator \#1 solution.

The results presented in Table 4 document the reproducibility of manufacturing calibrator solutions. A total of seven sets of calibrator solutions were prepared separately by two different technicians and each component of each lot, along with the admixture of the components, was then evaluated in quadruplicate using varying concentrations of anti-IgG-biotin. The overall average \% CV was calculated to be $10.4 \%$ (range, $3.5 \%-18.8 \%$ ) for the dog calibrator component and $9.8 \%$ (range, $3.1 \%-20.1 \%$ ) for the cat calibrator component; the admixture yielded an overall \% CV of $12.8 \%$ (range, $3.4 \%-19.6 \%)$. It is noteworthy that the signals generated at the projected assay concentration $(25 \mathrm{nG} / \mathrm{mL})$ are reduced in direct proportion to the calibrator dilution; a three-fold dilution in sera results in an approximate two-fold reduction in signal.

To define the overall reproducibility of the antigen specific IgG ELISA, the calibrator solutions were evaluated by two separate technicians on multiple occasions using grass pollen extract coated wells. The results presented in Table 5 document the overall \% CV calculated from observed results within multiple assay runs performed by two separate operators. The overall average $\mathrm{OD}_{405}$ calculated for the five reactive calibrators and expressed as milli-absorbance units was
$3099,1591,648,254$, and 139 for calibrator \# 1 - 5, respectively; the average background response was calculated to be 78 milli-absorbance units. The average intra-assay \% CV among positive calibrators (\#1-5) was calculated to be $5.7 \%$ (range $1.3 \%$ - 12.9\%); substantial differences between operators and assay runs were not detected. To be expected, the greatest intra-assay variability was evident with the background ODs (average 8.3; range $3.2-19.2 \%$ ); these responses are well within the expected limits for assays of this sort $[2,16,17]$ The average interassay variance for each of the operators was indistinguishable and the average \% CV was calculated to be $10.6 \%$ (range $7.0-14.6 \%$ ). The intra-laboratory \% CV among reactive calibrators (\#1-5) remained relatively constant (average $11.8 \%$; range $7.4-13.1 \%$ ), while the background variance was calculated to be only slightly higher at $14.0 \%$.

To document the utility of the dual assay for detection of antigen specific IgG in the sera of dogs and cats, 59 samples from each species were evaluated on a panel of pollen and environmental antigens. In addition, 54 samples of each species were evaluated on a panel of food extracts. Preliminary evaluations (data not shown) indicate that antigen specific IgG is present at varying levels in the sera of essentially all dogs and cats, and dilution of each sera sample is necessary to estimate the relative quantities of antigen specific IgG. The results presented in Table 6 demonstrate that the magnitude of responses evident in dog sera spans the range of reactivity detectable using the antigen specific IgG ELISA following dilution of 1: 3000 . Although the range of EAU detected with the sera samples varies among different antigens tested, the overall response indicates that approximately $30 \%$ of the sample responses are within the lower range of detection (0$1000 \mathrm{EAU}$ ) and approximately $20 \%$ of the sample response are in the upper range of detection (3001-4000 EAU), whereas nearly $50 \%$ of all responses are with the mid-range of detection (1001-3000 EAU). A similar response profile is also evident with cat sera samples (Table 7) following a dilution of 1: 3000 , and approximately $50 \%$ of all sample responses fall within the mid-range of detectability. However, it appears that a greater percentage of samples (36\%) yield responses in the upper range of detection. Whether or not this observation is actually due to a greater quantity of antigen specific IgG in cat sera or merely a function of the population of samples selected for evaluation remains to be determined. However, in light of the quite similar responses evident for dogs (Table 8) and cats (Table 9) with the food antigen panel it is tempting to speculate that the differences observed with the pollen and environmental antigens is actually a function of the population selection. 
Table 4. Reactivity of multiple manufacture lots of calibrator solutions detected with admixtures of anti-dog IgG-biotin and anti-cat IgG-biotin.

\begin{tabular}{|c|c|c|c|c|c|c|c|}
\hline \multirow{2}{*}{$\begin{array}{l}\text { Anti-lgG-Biotin* } \\
\text { (nG/mL) }\end{array}$} & \multirow{2}{*}{$\begin{array}{c}\mathrm{N}^{+} \\
(=28) \\
\end{array}$} & \multicolumn{5}{|c|}{ Dog Calibrators $^{\ddagger}$} & \multirow{2}{*}{$\underline{B G}$} \\
\hline & & $\# 1$ & $\# 2$ & \#3 & $\# 4$ & $\# 5$ & \\
\hline \multirow[t]{2}{*}{50} & Average & 3.335 & 2.085 & 0.875 & 0.362 & 0.175 & 0.078 \\
\hline & $\% \mathrm{CV}$ & 3.5 & 12.4 & 12.4 & 10.2 & 8.5 & 5.8 \\
\hline \multirow[t]{2}{*}{25} & Average & 2.951 & 1.494 & 0.632 & 0.284 & 0.146 & 0.067 \\
\hline & $\% \mathrm{CV}$ & 8.7 & 18.8 & 16.8 & 11.4 & 10.5 & 12.6 \\
\hline \multirow[t]{2}{*}{12.5} & Average & 2.525 & 1.768 & 0.707 & 0.301 & 0.142 & 0.064 \\
\hline & $\% \mathrm{CV}$ & 14.9 & 8.2 & 7.1 & 6.1 & 6.3 & 6.9 \\
\hline \multirow{3}{*}{$\begin{array}{c}\text { Anti-lgG-Biotin* } \\
(\underline{\mathrm{nG} / \mathrm{mL})}\end{array}$} & $\mathrm{N}^{\dagger}$ & \multicolumn{5}{|c|}{ Cat Calibrators $^{\ddagger}$} & \multirow{2}{*}{$\underline{B G}$} \\
\hline & $(=28)$ & $\# 1$ & $\# 2$ & \#3 & $\# 4$ & $\# 5$ & \\
\hline & Average & 3.371 & 2.108 & 0.880 & 0.357 & 0.171 & 0.076 \\
\hline 50 & $\% \mathrm{CV}$ & 3.1 & 12.3 & 11.9 & 9.3 & 7.0 & 7.1 \\
\hline \multirow[t]{2}{*}{25} & Average & 3.265 & 1.830 & 0.760 & 0.307 & 0.151 & 0.070 \\
\hline & $\% \mathrm{CV}$ & 6.7 & 17.1 & 20.1 & 12.9 & 9.9 & 10.5 \\
\hline \multirow[t]{2}{*}{12.5} & Average & 2.868 & 1.353 & 0.559 & 0.242 & 0.132 & 0.075 \\
\hline & $\% \mathrm{CV}$ & 9.2 & 9.9 & 7.0 & 5.9 & 4.7 & 4.7 \\
\hline \multirow{2}{*}{$\begin{array}{c}\text { Anti-lgG-Biotin* } \\
(\underline{\mathrm{nG} / \mathrm{mL})}\end{array}$} & $\mathrm{N}^{+}$ & \multicolumn{5}{|c|}{ Dog / Cat Calibrators ${ }^{\ddagger}$} & \multirow{2}{*}{$\underline{B G}$} \\
\hline & $(=28)$ & $\# 1$ & $\# 2$ & $\# 3$ & $\# 4$ & $\# 5$ & \\
\hline \multirow[t]{2}{*}{50} & Average & 3.338 & 2.101 & 0.891 & 0.362 & 0.177 & 0.077 \\
\hline & $\% \mathrm{CV}$ & 3.4 & 13.5 & 14.5 & 11.9 & 8.4 & 4.4 \\
\hline \multirow[t]{2}{*}{25} & Average & 3.206 & 1.794 & 0.736 & 0.303 & 0.150 & 0.068 \\
\hline & $\% \mathrm{CV}$ & 7.8 & 19.6 & 19.3 & 16.5 & 11.3 & 9.3 \\
\hline \multirow[t]{2}{*}{12.5} & Average & 2.595 & 1.248 & 0.515 & 0.220 & 0.121 & 0.072 \\
\hline & $\% \mathrm{CV}$ & 14.5 & 16.8 & 12.5 & 12.1 & 9.7 & 3.9 \\
\hline
\end{tabular}

*Final concentration of anti-dog IgG-biotin and anti-cat IgG-biotin in equal concentration admixtures. †Seven separate lots of each set of calibrators was prepared by two different operators and each calibrator solution was evaluated in quadruplicate in grass pollen allergen coated wells. Dog calibrators are prepared using dog sera only, cat calibrators are prepared using cat sera only, and Dog/Cat Calibrators contain both dog and cat sera.

:Calibrator \#1 is prepared as a dilution of a sera pool that is highly reactive to grass pollen allergens; calibrators \#2 - \#5 are prepared as a serial 3-fold dilution of calibrator \#1.

*Background responses observed with diluent in place of serum sample. 
Table 5. Assay variance of IgG ELISA calibrator solutions observed with different laboratory runs by different operators.

\begin{tabular}{|c|c|c|c|c|c|c|c|c|}
\hline \multirow{2}{*}{ Variance } & \multirow{2}{*}{$\underline{\text { Trial }}$} & \multirow{2}{*}{$\underline{N}$} & \multicolumn{5}{|c|}{ Calibrator \% CV } & \multirow{2}{*}{$\begin{array}{l}\mathrm{BG}^{\dagger} \\
\% \mathrm{CV} \\
\end{array}$} \\
\hline & & & $\# 1$ & $\# 2$ & $\underline{\# 3}$ & $\underline{\# 4}$ & $\underline{\# 5}$ & \\
\hline \multicolumn{8}{|l|}{ Inter-Assay } & 14.0 \\
\hline Operator \# 1 & & 336 & 7.8 & 13.6 & 14.2 & 14.2 & 14.6 & 15.0 \\
\hline Operator \# 2 & & 336 & 7.0 & 11.8 & 12.1 & 11.3 & 10.9 & 13.0 \\
\hline \multicolumn{9}{|l|}{ Intra-Assay } \\
\hline \multirow{9}{*}{ Operator \#1 } & \# 1 & 32 & 3.2 & 4.5 & 5.4 & 6.7 & 6.0 & 8.4 \\
\hline & \# 2 & 32 & 1.3 & 4.2 & 2.2 & 2.5 & 3.9 & 7.3 \\
\hline & \# 3 & 32 & 2.2 & 8.0 & 12.1 & 12.9 & 9.4 & 19.2 \\
\hline & \# 4 & 8 & 2.9 & 3.0 & 3.8 & 3.0 & 3.0 & 3.4 \\
\hline & \# 5 & 8 & 1.9 & 2.6 & 4.7 & 9.2 & 2.9 & 4.0 \\
\hline & \# 6 & 8 & 2.4 & 4.1 & 2.5 & 3.1 & 1.7 & 3.6 \\
\hline & \# 7 & 32 & 2.9 & 9.2 & 12.2 & 11.2 & 12.8 & 15.7 \\
\hline & \# 8 & 40 & 4.8 & 10.4 & 8.7 & 6.2 & 8.3 & 10.5 \\
\hline & \# 9 & 144 & 6.4 & 10.1 & 10.8 & 10.6 & 8.2 & 7.1 \\
\hline \multirow{9}{*}{ Operator \#2 } & \# 1 & 32 & 2.1 & 7.0 & 6.0 & 6.1 & 5.9 & 6.6 \\
\hline & \# 2 & 32 & 3.1 & 3.4 & 4.4 & 6.0 & 7.4 & 9.3 \\
\hline & \# 3 & 32 & 1.9 & 2.4 & 3.6 & 7.0 & 5.3 & 8.1 \\
\hline & \# 4 & 8 & 2.5 & 3.2 & 4.8 & 3.0 & 1.9 & 4.1 \\
\hline & \# 5 & 8 & 3.0 & 5.2 & 4.5 & 4.3 & 2.2 & 3.2 \\
\hline & \# 6 & 8 & 1.8 & 4.8 & 7.3 & 5.4 & 5.1 & 3.5 \\
\hline & \# 7 & 32 & 1.9 & 4.9 & 6.6 & 7.3 & 10.6 & 18.2 \\
\hline & \# 8 & 40 & 5.6 & 8.8 & 8.6 & 7.2 & 6.9 & 9.6 \\
\hline & \# 9 & 144 & 6.8 & 11.4 & 12.5 & 9.0 & 6.3 & 6.5 \\
\hline
\end{tabular}

*Calibrator \#1 is prepared as a dilution of a sera pool that is highly reactive to grass pollen allergens; calibrators \#2 - \#5 are prepared as a serial 3-fold dilution of calibrator \#1.

$\uparrow$ Background responses observed with diluent in place of serum sample. 
Table 6. Range of ELISA reactivity observed with randomly selected DOG sera samples when evaluated against a panel of pollen allergens using an ELISA specific for dog IgG and cat IgG.

\begin{tabular}{|c|c|c|c|c|c|c|c|c|}
\hline \multirow{3}{*}{ Allergens } & \multicolumn{8}{|c|}{ EAU* Range of Reactivity $^{*}$} \\
\hline & \multicolumn{2}{|c|}{$0-1000$} & \multicolumn{2}{|c|}{$1001-2000$} & \multicolumn{2}{|c|}{$2001-3000$} & \multicolumn{2}{|c|}{$3001-4000$} \\
\hline & $\mathrm{N}$ & $\%$ Total & $\mathrm{N}$ & $\%$ Total & $\mathrm{N}$ & $\%$ Total & $\mathrm{N}$ & $\%$ Total \\
\hline \multicolumn{9}{|l|}{ Mites } \\
\hline Acaris siro & 23 & 39.0 & 17 & 28.8 & 10 & 16.9 & 9 & 15.3 \\
\hline Dermatophagoides farinae & 11 & 18.6 & 22 & 37.3 & 13 & 22.0 & 13 & 22.0 \\
\hline Dermatophagoides pteronyssinus & 16 & 27.1 & 22 & 37.3 & 13 & 22.0 & 8 & 13.6 \\
\hline Lepidoglyphus destructor & 16 & 27.1 & 22 & 37.3 & 10 & 16.9 & 11 & 18.6 \\
\hline Tyrophagus putrescentiae & 13 & 22.0 & 21 & 35.6 & 11 & 18.6 & 14 & 23.7 \\
\hline \multicolumn{9}{|l|}{ Grasses } \\
\hline June Grass (Poa pratensis) & 20 & 33.9 & 17 & 28.8 & 11 & 18.6 & 11 & 18.6 \\
\hline Meadow fescue (Festuca pratensis) & 22 & 37.3 & 19 & 32.2 & 7 & 11.9 & 11 & 18.6 \\
\hline Orchard Grass (Dactylis glomerata) & 18 & 30.5 & 16 & 27.1 & 12 & 20.3 & 13 & 22.0 \\
\hline Perennial Rye (Lolium perenne) & 22 & 37.3 & 17 & 28.8 & 10 & 16.9 & 10 & 16.9 \\
\hline \multicolumn{9}{|l|}{ Trees } \\
\hline Birch (Betula pendula) & 16 & 27.1 & 17 & 28.8 & 15 & 25.4 & 11 & 18.6 \\
\hline Cypress (Cupressus sempervirens) & 44 & 74.6 & 9 & 15.3 & 3 & 5.1 & 3 & 5.1 \\
\hline Hazelnut (Corylus avellana) & 20 & 33.9 & 17 & 28.8 & 13 & 22.0 & 9 & 15.3 \\
\hline Olive (Olea europaea) & 17 & 28.8 & 20 & 33.9 & 9 & 15.3 & 13 & 22.0 \\
\hline Populus mix (P. nigra, P. tremula, P. alba) & 17 & 28.8 & 18 & 30.5 & 11 & 18.6 & 13 & 22.0 \\
\hline Willow Black (Salix discolor) & 19 & 32.2 & 21 & 35.6 & 11 & 18.6 & 8 & 13.6 \\
\hline \multicolumn{9}{|l|}{ Weeds } \\
\hline English Plantain (Plantago lanceolata) & 20 & 33.9 & 12 & 20.3 & 15 & 25.4 & 12 & 20.3 \\
\hline Lambs Quarter (Chenopodium album) & 21 & 35.6 & 20 & 33.9 & 8 & 13.6 & 10 & 16.9 \\
\hline Mugwort (Artemisia vulgaris) & 23 & 39.0 & 18 & 30.5 & 11 & 18.6 & 7 & 11.9 \\
\hline Pellitory (Parietaria officinalis ) & 15 & 25.4 & 20 & 33.9 & 14 & 23.7 & 10 & 16.9 \\
\hline Ragweed (Ambrosia trifida, A. artemisiifolia) & 29 & 49.2 & 14 & 23.7 & 10 & 16.9 & 6 & 10.2 \\
\hline Sheep Sorrel (Rumex acetosella) & 19 & 32.2 & 19 & 32.2 & 11 & 18.6 & 10 & 16.9 \\
\hline \multicolumn{9}{|l|}{ Fungi } \\
\hline Alternaria alternata & 2 & 3.4 & 9 & 15.3 & 11 & 18.6 & 37 & 62.7 \\
\hline Aspergillus fumagatis & 6 & 10.2 & 12 & 20.3 & 15 & 25.4 & 26 & 44.1 \\
\hline Cladosporium herbarum & 5 & 8.5 & 9 & 15.3 & 11 & 18.6 & 34 & 57.6 \\
\hline Overall & 434 & 30.6 & 408 & 28.8 & 265 & 18.7 & 309 & 21.8 \\
\hline
\end{tabular}

*EAU = ELISA Absorbance Units, which were calculated as the OD in milli-absorbance units corrected for background (Sample OD - Background OD x 1000) and normalized to a four-point calibration curve. 
Table 7. Range of ELISA reactivity observed with randomly selected CAT sera samples when evaluated against a panel of pollen allergens using an ELISA specific for dog IgG and cat IgG.

\begin{tabular}{|c|c|c|c|c|c|c|c|c|}
\hline \multirow{3}{*}{$\underline{\text { Allergens }}$} & \multicolumn{8}{|c|}{ EAU* Range of Reactivity } \\
\hline & \multicolumn{2}{|c|}{$0-1000$} & \multicolumn{2}{|c|}{$1001-2000$} & \multicolumn{2}{|c|}{$2001-3000$} & \multicolumn{2}{|c|}{$3001-4000$} \\
\hline & $\mathrm{N}$ & $\%$ Total & $\mathrm{N}$ & $\%$ Total & $\mathrm{N}$ & $\%$ Total & $\mathrm{N}$ & $\%$ Total \\
\hline \multicolumn{9}{|l|}{ Mites } \\
\hline Acaris siro & 8 & 13.6 & 12 & 20.3 & 13 & 22.0 & 26 & 44.1 \\
\hline Dermatophagoides farinae & 3 & 5.1 & 12 & 20.3 & 15 & 25.4 & 29 & 49.2 \\
\hline Dermatophagoides pteronyssinus & 5 & 8.5 & 9 & 15.3 & 22 & 37.3 & 23 & 39.0 \\
\hline Lepidoglyphus destructor & 10 & 16.9 & 17 & 28.8 & 16 & 27.1 & 16 & 27.1 \\
\hline Tyrophagus putrescentiae & 4 & 6.8 & 13 & 22.0 & 11 & 18.6 & 31 & 52.5 \\
\hline \multicolumn{9}{|l|}{ Grasses } \\
\hline June Grass (Poa pratensis ) & 9 & 15.3 & 18 & 30.5 & 19 & 32.2 & 13 & 22.0 \\
\hline Meadow fescue (Festuca pratensis) & 15 & 25.4 & 22 & 37.3 & 8 & 13.6 & 14 & 23.7 \\
\hline Orchard Grass (Dactylis glomerata) & 8 & 13.6 & 19 & 32.2 & 12 & 20.3 & 20 & 33.9 \\
\hline Perennial Rye (Lolium perenne) & 18 & 30.5 & 21 & 35.6 & 7 & 11.9 & 13 & 22.0 \\
\hline \multicolumn{9}{|l|}{ Trees } \\
\hline Birch (Betula pendula) & 10 & 16.9 & 16 & 27.1 & 7 & 11.9 & 26 & 44.1 \\
\hline Cypress (Cupressus sempervirens) & 21 & 35.6 & 15 & 25.4 & 13 & 22.0 & 10 & 16.9 \\
\hline Hazelnut (Corylus avellana) & 13 & 22.0 & 22 & 37.3 & 12 & 20.3 & 12 & 20.3 \\
\hline Olive (Olea europaea) & 7 & 11.9 & 20 & 33.9 & 12 & 20.3 & 20 & 33.9 \\
\hline Populus mix (P. nigra, P. tremula, P. alba) & 4 & 6.8 & 18 & 30.5 & 11 & 18.6 & 26 & 44.1 \\
\hline Willow Black (Salix discolor) & 8 & 13.6 & 19 & 32.2 & 17 & 28.8 & 15 & 25.4 \\
\hline \multicolumn{9}{|l|}{ Weeds } \\
\hline English Plantain (Plantago lanceolata) & 2 & 3.4 & 9 & 15.3 & 7 & 11.9 & 41 & 69.5 \\
\hline Lambs Quarter (Chenopodium album) & 2 & 3.4 & 5 & 8.5 & 9 & 15.3 & 43 & 72.9 \\
\hline Mugwort (Artemisia vulgaris) & 8 & 13.6 & 27 & 45.8 & 9 & 15.3 & 15 & 25.4 \\
\hline Pellitory (Parietaria officinalis ) & 3 & 5.1 & 12 & 20.3 & 11 & 18.6 & 33 & 55.9 \\
\hline Ragweed (Ambrosia trifida, A. artemisiifolia) & 23 & 39.0 & 17 & 28.8 & 10 & 16.9 & 9 & 15.3 \\
\hline Sheep Sorrel (Rumex acetosella) & 9 & 15.3 & 17 & 28.8 & 13 & 22.0 & 20 & 33.9 \\
\hline \multicolumn{9}{|l|}{ Fungi } \\
\hline Alternaria alternata & 6 & 10.2 & 18 & 30.5 & 17 & 28.8 & 18 & 30.5 \\
\hline Aspergillus fumagatis & 13 & 22.0 & 17 & 28.8 & 12 & 20.3 & 17 & 28.8 \\
\hline Cladosporium herbarum & 7 & 11.9 & 17 & 28.8 & 15 & 25.4 & 20 & 33.9 \\
\hline Overall & 216 & 15.3 & 392 & 27.7 & 298 & 21.0 & 510 & 36.0 \\
\hline
\end{tabular}

*EAU $=$ ELISA Absorbance Units, which were calculated as the OD in milli-absorbance units corrected for background (Sample OD - Background OD x 1000) and normalized to a four-point calibration curve. 
Table 8. Range of ELISA reactivity observed with randomly selected DOG sera samples when evaluated against a panel of food allergens using an ELISA specific for dog IgG and cat IgG.

\begin{tabular}{|c|c|c|c|c|c|c|c|c|}
\hline \multirow{3}{*}{ Allergens } & \multicolumn{8}{|c|}{ EAU* Range of Reactivity } \\
\hline & \multicolumn{2}{|c|}{$0-1000$} & \multicolumn{2}{|c|}{$1001-2000$} & \multicolumn{2}{|c|}{$2001-3000$} & \multicolumn{2}{|c|}{$3001-4000$} \\
\hline & $\mathrm{N}$ & $\%$ Total & $\mathrm{N}$ & $\%$ Total & $\mathrm{N}$ & $\%$ Total & $\mathrm{N}$ & $\%$ Total \\
\hline \multicolumn{9}{|l|}{ Meats } \\
\hline Beef & 8 & 14.8 & 21 & 38.9 & 8 & 14.8 & 17 & 31.5 \\
\hline Chicken & 22 & 40.7 & 24 & 44.4 & 5 & 9.3 & 3 & 5.6 \\
\hline Duck & 20 & 37.0 & 22 & 40.7 & 9 & 16.7 & 3 & 5.6 \\
\hline Fish Mix & 30 & 55.6 & 15 & 27.8 & 6 & 11.1 & 3 & 5.6 \\
\hline Lamb & 16 & 29.6 & 26 & 48.1 & 6 & 11.1 & 6 & 11.1 \\
\hline Ostrich & 17 & 31.5 & 22 & 40.7 & 12 & 22.2 & 3 & 5.6 \\
\hline Pork & 23 & 42.6 & 24 & 44.4 & 4 & 7.4 & 3 & 5.6 \\
\hline Rabbit & 19 & 35.2 & 22 & 40.7 & 8 & 14.8 & 5 & 9.3 \\
\hline Salmon & 24 & 44.4 & 16 & 29.6 & 10 & 18.5 & 4 & 7.4 \\
\hline Tuna & 14 & 25.9 & 21 & 38.9 & 10 & 18.5 & 9 & 16.7 \\
\hline Turkey & 25 & 46.3 & 24 & 44.4 & 3 & 5.6 & 2 & 3.7 \\
\hline Venison & 19 & 35.2 & 18 & 33.3 & 7 & 13.0 & 10 & 18.5 \\
\hline \multicolumn{9}{|l|}{ Grains } \\
\hline Barley & 18 & 33.3 & 16 & 29.6 & 9 & 16.7 & 11 & 20.4 \\
\hline Corn & 7 & 13.0 & 18 & 33.3 & 18 & 33.3 & 11 & 20.4 \\
\hline Millet & 33 & 61.1 & 11 & 20.4 & 6 & 11.1 & 4 & 7.4 \\
\hline Oat & 49 & 90.7 & 4 & 7.4 & 0 & 0.0 & 1 & 1.9 \\
\hline Rice & 16 & 29.6 & 16 & 29.6 & 11 & 20.4 & 11 & 20.4 \\
\hline Wheat & 22 & 40.7 & 14 & 25.9 & 8 & 14.8 & 10 & 18.5 \\
\hline \multicolumn{9}{|l|}{ Other } \\
\hline Beet Pulp & 36 & 66.7 & 12 & 22.2 & 4 & 7.4 & 2 & 3.7 \\
\hline Brewer's Yeast & 4 & 7.4 & 8 & 14.8 & 13 & 24.1 & 29 & 53.7 \\
\hline Egg & 46 & 85.2 & 7 & 13.0 & 1 & 1.9 & 0 & 0.0 \\
\hline Milk & 22 & 40.7 & 18 & 33.3 & 7 & 13.0 & 7 & 13.0 \\
\hline Potato & 16 & 29.6 & 21 & 38.9 & 11 & 20.4 & 6 & 11.1 \\
\hline Soybean & 18 & 33.3 & 17 & 31.5 & 12 & 22.2 & 7 & 13.0 \\
\hline Overall & 524 & 40.4 & 417 & 32.2 & 188 & 14.5 & 167 & 12.9 \\
\hline
\end{tabular}

*EAU = ELISA Absorbance Units, which were calculated as the OD in milli-absorbance units corrected for background (Sample OD - Background OD x 1000) and normalized to a four-point calibration curve. 
Table 9. Range of ELISA reactivity observed with randomly selected CAT sera samples when evaluated against a panel of food allergens using an ELISA specific for dog IgG and cat IgG.

\begin{tabular}{|c|c|c|c|c|c|c|c|c|}
\hline \multirow{3}{*}{ Allergens } & \multicolumn{8}{|c|}{ EAU* Range of Reactivity } \\
\hline & \multicolumn{2}{|c|}{$0-1000$} & \multicolumn{2}{|c|}{$1001-2000$} & \multicolumn{2}{|c|}{$2001-3000$} & \multicolumn{2}{|c|}{$3001-4000$} \\
\hline & $\mathrm{N}$ & $\%$ Total & $\mathrm{N}$ & $\%$ Total & $\mathrm{N}$ & $\%$ Total & $\mathrm{N}$ & $\%$ Total \\
\hline \multicolumn{9}{|l|}{ Meats } \\
\hline Beef & 9 & 16.7 & 7 & 13.0 & 11 & 20.4 & 27 & 50.0 \\
\hline Chicken & 33 & 61.1 & 12 & 22.2 & 7 & 13.0 & 2 & 3.7 \\
\hline Duck & 29 & 53.7 & 14 & 25.9 & 7 & 13.0 & 4 & 7.4 \\
\hline Fish Mix & 41 & 75.9 & 9 & 16.7 & 2 & 3.7 & 2 & 3.7 \\
\hline Lamb & 12 & 22.2 & 21 & 38.9 & 9 & 16.7 & 12 & 22.2 \\
\hline Ostrich & 28 & 51.9 & 17 & 31.5 & 5 & 9.3 & 4 & 7.4 \\
\hline Pork & 26 & 48.1 & 16 & 29.6 & 7 & 13.0 & 5 & 9.3 \\
\hline Rabbit & 30 & 55.6 & 16 & 29.6 & 6 & 11.1 & 2 & 3.7 \\
\hline Salmon & 26 & 48.1 & 18 & 33.3 & 5 & 9.3 & 5 & 9.3 \\
\hline Tuna & 26 & 48.1 & 20 & 37.0 & 5 & 9.3 & 3 & 5.6 \\
\hline Turkey & 30 & 55.6 & 15 & 27.8 & 4 & 7.4 & 5 & 9.3 \\
\hline Venison & 15 & 27.8 & 12 & 22.2 & 11 & 20.4 & 16 & 29.6 \\
\hline \multicolumn{9}{|l|}{ Grains } \\
\hline Barley & 6 & 11.1 & 28 & 51.9 & 10 & 18.5 & 10 & 18.5 \\
\hline Corn & 6 & 11.1 & 17 & 31.5 & 15 & 27.8 & 16 & 29.6 \\
\hline Millet & 31 & 57.4 & 15 & 27.8 & 3 & 5.6 & 5 & 9.3 \\
\hline Oat & 42 & 77.8 & 10 & 18.5 & 2 & 3.7 & 0 & 0.0 \\
\hline Rice & 7 & 13.0 & 20 & 37.0 & 12 & 22.2 & 15 & 27.8 \\
\hline Wheat & 13 & 24.1 & 17 & 31.5 & 13 & 24.1 & 11 & 20.4 \\
\hline \multicolumn{9}{|l|}{ Other } \\
\hline Beet Pulp & 22 & 40.7 & 18 & 33.3 & 5 & 9.3 & 9 & 16.7 \\
\hline Brewer's Yeast & 12 & 22.2 & 20 & 37.0 & 12 & 22.2 & 10 & 18.5 \\
\hline Egg & 37 & 68.5 & 12 & 22.2 & 3 & 5.6 & 2 & 3.7 \\
\hline Milk & 16 & 29.6 & 8 & 14.8 & 12 & 22.2 & 18 & 33.3 \\
\hline Potato & 16 & 29.6 & 20 & 37.0 & 10 & 18.5 & 8 & 14.8 \\
\hline Soybean & 8 & 14.8 & 24 & 44.4 & 8 & 14.8 & 14 & 25.9 \\
\hline Overall & 521 & 40.2 & 386 & 29.8 & 184 & 14.2 & 205 & 15.8 \\
\hline
\end{tabular}

*EAU $=$ ELISA Absorbance Units, which were calculated as the OD in milli-absorbance units corrected for background (Sample OD - Background OD x 1000) and normalized to a four-point calibration curve. 
The results presented in Table 8 and Table 9 document the food antigen specific IgG reactivity detected in dogs and cats, respectively. Overall, approximately $40 \%$ of the samples in both dogs and cats tested for food specific IgG yielded responses in the lower range of detectability $(0-1000 \mathrm{EAU})$; whereas, the percentage of results in the upper range of detection (>3001 EAU) was $12.9 \%$ for dogs samples and $15.8 \%$ for cat samples. Consistent with the results for the pollen specific IgG detection, approximately $50 \%$ of the samples tested yielded results within the mid-range of detection. In spite of the skewed response to food antigens, these results combined with the results for pollen and environmental antigen document the utility of the single ELISA for detection of antigen specific IgG in both dogs and cats.

\section{Discussion}

We have previously optimized and characterized an enzyme-linked immunosorbent assay (ELISA) for detection of allergen specific IgE in dogs [2]. This ELISA effectively serves as the prototype for developing similar assays for detection of antigen specific immunoglobulins of various isotypes in different species of companion animals. Logic dictates that when the ELISA protocol for detection of isotype specific immunoglobulins are maintained, the majority of assay components become interchangeable among assays. The only components that are unique to any immunoglobulin specific ELISA of this sort are the biotinylated secondary anti- $\operatorname{IgX}$ antibodies and the assay specific calibrators and control reagents. Thus, characterizing these species specific components should yield an optimized assay for that specific immunoglobulin isotype for any given animal companion. Furthermore, the results yielded will remain of the same sort and be easily accommodated by any reporting convention.

A critical component of any laboratory test is the validity of such a test (i.e. Does the test correlate with a disease state or condition?). However, before the validity of a test can be determined, it is necessary to ensure that the laboratory test is reliable. In determining reliability it is important to document reproducible manufacture of the assay components and of the reproducibility of the results generated among assay runs. In the current evaluation, we characterize a single ELISA for detection of antigen specific IgG not only in dogs but in cats as well. We have defined the procedure to reproducibly manufacture the critical components of the proposed assay and we document the reproducibility of the assay on multiple occasions. In addition, we have evaluated the relative levels of antigen specific IgG in dogs and cats to multiple environmental and food antigens using this assay.

In light of the observation that the majority of cats and dogs possess antigen specific IgG in their serum to essentially "all" antigens [8-11] and a serum dilution of 1: 3000 is required in the current assay to reach a response level that falls within the range of a calibration curve, the concept of "assay cutoff" loses meaning. For assays of this sort, the concept of lower limits of detection takes on more meaning. Considering the results presented in Table 4 which demonstrate that a three-fold dilution in sera results in an approximate two-fold reduction in signal and that the signal evident for calibrator \# 5 (calculated to be 0.152 .) is approximately twice the background response (calculated to be 0.072 ), it appears that a lower limit is defined by calibrator \# 5 at 0.150 (i.e. $150 \mathrm{EAU}$ ). This lower limit of detection value is akin to the cutoff value defined for our macELISA for detection of allergen specific IgE in dogs and cats [2].

The IgG results might be reported in ELISA absorbance Units (EAU), which are merely background corrected responses normalized to the calibration curve and expressed in milli-absorbance. The daily results can then be normalized to historical observations of responses observed with the calibrator solutions, which will then allow direct comparison of results collected within different daily assay runs. This form of reporting will be in accord with the macELISA reporting for detection of allergen specific IgE [2].

Results might also be presented in a categorical representation of the relative quantity of antigen specific $\operatorname{IgG}[16,17]$. To determine the Relative IgG Units (RGU) will require interpolation from a regression curve (preferably a 4-parameter regression curve) created by plotting the background corrected optical density observed with each of the calibrators versus an arbitrarily assigned concentration value (perhaps 100,000 for calibrator \#1) based upon the dilution schema used for preparing the calibrator solutions. The dose response curve evident with the calibrator solutions (Table 4) indicates that a three-fold dilution of serum results in an approximate two-fold reduction in signals generated. Thus, the relative amount of antigen specific IgG that might be detected at the lower limit of detection (150 EAU) will need to be increased three-fold to yield a response of 300 EAU and nine-fold to yield a response of $600 \mathrm{EAU}$; to generate a maximal signal (4000 EAU) will require nearly a 150 -fold increase in antigen specific IgG. These finding then provide the bases for categorizing EAU responses into RGU as depicted in Table 10. Reporting of results in quantitative terms must await the characterization of known quantities of affinity purified antigen specific IgG (for each antigen of interest) that can be incorporated into a standard curve which can replace the calibration curve of the current assay.

Clinical improvement following immunotherapy is not simply due to increased production of IgG specific for the components in the treatment therapy, but it has been shown that the level of antigen specific IgG increases in the sera of dogs that have received an immunotherapeutic regime of allergen injections [18]. It is presumed that the IgG acts as a "blocking antibody" against antigens involved in IgE mediated allergy. Although improved responses due to allergen specific immunotherapy (ASIT) are likely a result of active modulation of the immune regulation mechanism, [9, 19-21 ] monitoring of the level of antigen specific IgG following immunotherapy might be included as an adjunct tool to insure the well-being of an allergic animal that has been placed on an immunotherapeutic allergen regime [18]. The assay characterized herein is readily adaptable to such a function.

Adverse food reactions in companion animals can be either food allergy or food intolerance. Allergy has an immunologic basis for its pathogenesis; but an immunologic basis for food intolerance has not be documented $[9,19-21]$. The current methods for detection of food allergy includes elimination and challenge diets and serum testing might then be useful for selection of foods that might be included in an elimination-challenge diet. Commercial tests are available for detection of food-specific IgE or IgG in serum, and are promoted as diagnostic tools for food intolerance. Yet, the clinical sensitivity and specificity (validity) of these assays have not yet been adequately documented. 
Table 10. Relative categories of IgG in relation to EAU and RGU and interpretation of results.

\section{Category /Class}

Negative

1

2

3

4
EAU* Range

$0-150$

$151-300$

$301-600$

601-1200

$1200-2400$

$>2400$
$\underline{\mathrm{RGU}^{\dagger}}$

0

$\geq 1.0$

$\geq 3$

$\geq 9$

$\geq 27$

$\geq 81$
Interpretation

No IgG Detectable

Equivocal

Positive<smiles>C=C</smiles>

Increasing levels of allergen specific IgG

5

*EAU $=$ ELISA Absorbance Units, which were calculated as the OD in milli-absorbance units corrected for background (Sample OD - Background OD x 1000) and normalized to a four-point calibration curve.

${ }^{\dagger} \mathrm{RGU}=$ Relative IgG Units interpolated from regression curve constructed from calibrator responses.

When considering IgG specific responses, high circulating levels of IgG are purported to correlate with clinical food allergy signs and detecting high levels or IgG to certain antigens is supposedly useful in pinpointing various antigens to avoid. Unfortunately, no well controlled prospective studies have been reported to support this contention. In light of the results presented herein (Tables 8-9), which document that the majority of cats and dogs contain IgG to most food stuffs, the mere detection of IgG specific for the food components cannot be the basis for allergy or intolerance. Thus, to conclude an involvement of IgG would require that a difference in the level of IgG to a specific antigen be finite or that a subclass of IgG is the causative agent. The authors are unaware of any study that defines the level of food specific IgG or subclass of IgG required to induce an allergic response. However, the development and characterization of the antigen specific IgG ELISA defined herein opens the possibilities for investigations in this arena. The results presented herein characterize a single ELISA that is capable of detecting antigen specific $\operatorname{IgG}$ in both dogs and cats. We have documented the reproducibility and robustness of the assay and defined its utility in detecting IgG specific for a number of different environmental and food antigens. Finally, we have documented the IgG reactivity to these antigen arrays in a subpopulation of dogs and cats. Collectively, these results provide a foundation for future studies intended to address the issues associated with the validity of IgG testing (i.e. clinical sensitivity and specificity) for various antigens, especially those contained in food stuffs.

\section{Authors Contributions}

Brennan McKinney and Karen Blankenship manufactured all components used throughout the evaluation and contributed to acquisition, analysis, and interpretation of data. Kenneth Lee contributed to the conception and design of the study; contributed to the acquisition, analysis, and interpretation of data; and drafted the manuscript. All authors gave final approval and agree to be accountable for all aspects of the work in ensuring that questions relating to the accuracy or integrity of any part of the work are appropriately investigated and resolved.

\section{Declaration of conflicting interests}

All authors are employees at Stallergenes Greer

\section{Funding}

Funding for this study was provided by Stallergenes Greer.

\section{References}

1. DeBoer DJ, Hillier A (2001) The ACVD task force on canine atopic dermatitis (XVI): laboratory evaluation of dogs with atopic dermatitis with serum-based "allergy" tests. Vet Immunol Immunopathol 81: 277-87.

2. Lee KW, Blankenship KD, McCurry ZM, Esch RE, DeBoer DJ, et al. (2009) Performance characteristics of a monoclonal antibody cocktail-based ELISA for detection of allergen-specific IgE in dogs and comparison with a high affinity $\operatorname{IgE}$ receptor-based ELISA. Vet Dermatol 20: 157-164. [crossref]

3. Stedman K, Lee K, Hunter S, Rivoire B, McCall C, et al. (2001) Measurement of canine IgE using the alpha chain of the human high affinity IgE receptor. Vet Immunol Immunopathol 78: 349-355. [crossref]

4. Lee KW, Blankenship KD, McCurry ZM, et al. (2012) Reproducibility of a Monoclonal Antibody Cocktail Based ELISA for Detection of Allergen Specific IgE in Dogs: Proficiency Monitoring of macELISA in Six US and European Laboratories. Vet Immunol Immunopathol 148: 267-275.

5. Lee KW, Blankenship K, McKinney B, et al. (2015) Proficiency monitoring of monoclonal antibody cocktail-based enzyme-linked immunosorbent assay for detection of allergen-specific immunoglobulin E in dogs. Journal of Veterinary Diagnostic Investigation 27: 461-469.

6. Lee KW, Blankenship K, McKinney B, et al. (2017) Continued Proficiency Monitoring Of Monoclonal Antibody Cocktail-Based Enzyme-Linked Immunosorbent Assay for Detection of Allergen Specific Immunoglobulin E in Dogs - 2016. Integr J Vet Biosci 1: 1-10

7. Thom N, Favrot C, Failing K, et al. (2010) Intra- and interlaboratory variability of allergen-specific IgE levels in atopic dogs in three different laboratories using the 
Kenneth Lee (2018) Development and Characterization of a Single Enzyme Linked Immunosorbent Assay for Detection of Antigen Specific IgG in Both Dogs and Cats

Fc-??receptor testing. Vet Immunol Immunopathol 133: 183-189.

8. Halliwell, R.E.W., Gordon, C.M., Horvath, C. (2005) IgE and IgG antibodies to food antigens in sera from normal dogs, dogs with atopic dermatitis and dogs with adverse food reactions. In: Hillier, A., Foster, A.P., Kwochka, K.W. (Eds.), Advances in Veterinary Dermatology. Blackwell Publishing, Oxford 28-35.

9. Pucheu-Haston CM, Bizikova P, Eisenschenk MN, Santoro D, Nuttall T, et al. (2015) Review: The role of antibodies, autoantigens and food allergens in canine atopic dermatitis. Vet Dermatol 26: 115-115e30. [crossref]

10. Bethlehem S, Bexley J, Mueller RS (2012) Patch testing and allergen-specific serum IgE and IgG antibodies in the diagnosis of canine adverse food reactions. Vet Immunol Immunopathol 145: 582-589.

11. Zimmer A, Bexley J, Halliwell RE, et al. (2011) Food allergen-specific serum $\mathrm{IgG}$ and IgE before and after elimination diets in allergic dogs. Vet Immunol Immunopathol 144: 442-447

12. Hofmaier S, Comberiati P, Matricardi PM (2014) Immunoglobulin G in IgEmediated allergy and allergen-specific immunotherapy. Eur Ann Allergy Clin Immunol 46: 6-11. [crossref]

13. Jeffers JG, Shanley KJ, Meyer EK (1991) Diagnostic testing of dogs for food hypersensitivity. J Am Vet Med Assoc 198: 245-250. [crossref]

14. Hardy JI, Hendricks A, Loeffler A, et al. 2014. Food-specific serum IgE and IgG reactivity in dogs with and without skin disease: lack of correlation between laboratories. Vet Dermatol 25: 447-e70.

15. Foster AP, Knowles TG, Moore AH, Cousins PD, Day MJ, et al. (2003) Serum $\mathrm{IgE}$ and IgG responses to food antigens in normal and atopic dogs, and dogs with gastrointestinal disease. Vet Immunol Immunopathol 92: 113-124. [crossref]

16. Tijssen P (1993) Processing of data and reporting of results of enzyme immunoassays. In: Burdon, RH, van Knippenberg PH, editors. Practice and Theory of Enzyme Immunoassays 385-421. Elsevier, Amsterdam.

17. World Organisation for Animal Health (OIE) (2014) Development and optimization of antibody detection assays. In: Manual of Diagnostic Tests and Vaccines for Terrestrial Animals, OIE, Paris, France.

18. Hites MJ, Kleinbeck ML, Loker JL, Lee KW (1989) Effect of immunotherapy on the serum concentrations of allergen-specific $\operatorname{IgG}$ antibodies in dog sera. Vet Immunol Immunopathol 22: 39-51. [crossref]

19. Kennis RA (2006) Food allergies: update of pathogenesis, diagnoses, and management. Vet. Clin. North Am. Small Anim Pract 36: 175-184.

20. Akdis CA, Akdis M (2015) Mechanisms of allergen-specific immunotherapy and immune tolerance to allergens. World Allergy Organ J 2015; 8: 17

21. Mandigers P, German AJ (2010) Dietary hypersensitivity in cats and dogs. Tijdschr Diergeneeskd 135: 706-710. [crossref]

\section{Citation:}

Brennan McKinney, Karen Blankenship and Kenneth Lee (2018) Development and Characterization of a Single Enzyme Linked Immunosorbent Assay for Detection of Antigen Specific IgG in Both Dogs and Cats. Integr J Vet Biosci Volume 2(1): 1-15 